\title{
A importância da abordagem não-farmacológica da hipertensão arterial na Atenção Primária à Saúde
}

The importance of a nondrug approach to arterial hypertension in Primary Health Care

Carolina Fajardo ${ }^{1}$

\section{RESUMO}

A hipertensão arterial sistêmica (HAS) representa um sério problema de saúde no Brasil, tanto por sua prevalência elevada como por suas taxas de morbimortalidade. É uma patologia por si mesma e um fator de risco clássico para as doenças do aparelho circulatório, além de interferir diretamente na qualidade de vida das pessoas por ela acometidas e na de seus familiares. Medidas que possibilitem a sua prevenção e o seu controle devem ser implementadas. Um espaço ideal para a prática de medidas que proporcionem a prevenção e a detecção precoce da HAS é a Atenção Primária à Saúde, por meio da Estratégia Saúde da Família. Sua abordagem pode ser feita por meio de mudanças no estilo de vida e/ou de tratamento medicamentoso com drogas anti-hipertensivas. A adesão ao tratamento é necessária para o seu sucesso e a promoção de estratégias que a possibilitem deve ser uma meta da equipe de saúde. Neste trabalho, será dada ênfase ao tratamento não-farmacológico da HAS. Cada abordagem será apresentada e estudos epidemiológicos que comprovem o seu valor serão relatados.

\section{ABSTRACT}

Systemic arterial hypertension (SAH) represents a serious health problem in Brazil, not only for its high prevalence but also for its morbimortality rates. Besides being a disease in itself, it is a classic risk factor for cardiovascular disease and interferes directly with the quality of life of patients and their families. Measures for its prevention and control must thus be implemented. An ideal space for early detection of high blood pressure $(B P)$, prevention and control is Primary Health Care with its Family Health Program. SAH can be approached by promoting changes in the lifestyle and/or through pharmacological therapy with antihypertensive drugs. Treatment adherence is vital for its success and promoting strategies for allowing this to happen should be a goal of the health team. In this work, emphasis will be given to the nondrug treatment of SAH through modifications in the lifestyle. Each approach will be presented and its value will be shown in epidemiological studies.

\section{PALAVRAS-CHAVE:}

- Hipertensão;

- Atenção Primária à Saúde;

- Promoção da Saúde;
KEY-WORDS:

- Hypertension;

- Primary Health Care;

- Health Promotion.

${ }^{1}$ Médica de Família e Comunidade. Residência em Medicina de Família e Comunidade pela UERJ. Coordenação do Programa de Saúde da Família, SES - RJ 


\section{I - Introdução}

As doenças cardiovasculares, dentre elas os acidentes vasculares encefálicos (AVC) e o infarto agudo do miocárdio (IAM), representam um sério problema de saúde pública, pois constituem a principal causa de morbimortalidade da população brasileira. A hipertensão arterial sistêmica (HAS) é um dos seus principais fatores de risco, atingindo de $11 \%$ a $20 \%$ da população adulta com mais de 20 anos. Estudos epidemiológicos mostraram que os fatores de risco clássicos (obesidade, diabetes mellitus, hipertensão arterial sistêmica, dislipidemia e sedentarismo) são responsáveis por menos de $40 \%$ dos óbitos ocorridos por doenças do aparelho circulatório1.

Foi observado que a diminuição da mortalidade cardiovascular não foi precedida pelo controle dos fatores de risco cardiovasculares clássicos, mas, sim, pela redução da morbidade por doenças infecciosas. Portanto, existem evidências de que o controle das doenças cardiovasculares deve sofrer uma mudança em seus paradigmas. Além do controle dos fatores de risco cardiovasculares clássicos, estratégias que busquem a mudança das condições de vida das pessoas são necessárias, para o efetivo controle dos processos crônicos degenerativos, causando um maior impacto na redução da morbimortalidade cardiovascular, além do simples controle dos fatores de risco cardiovasculares clássicos ${ }^{2}$.

Para alcançarmos um dos objetivos principais da abordagem terapêutica das pessoas com hipertensão, que é a redução das taxas de morbidade e mortalidade, ações de prevenção, a detecção precoce e o controle adequado precisam ser implementados.

A Estratégia Saúde da Família (ESF), que constitui a política do Ministério da Saúde de reorientação da Atenção Primária à Saúde ${ }^{3}$, é o locus ideal para a prática dessas ações, sendo também um espaço privilegiado para o trabalho de prevenção e promoção da saúde, ou seja, a atuação em situações de risco para o adoecimento, antes que a doença esteja instalada. Além disso, a ESF possibilita o aumento do acesso ao sistema de saúde, condição importante para adesão ao tratamento.

A equipe de saúde da família tem, entre as suas funções e atribuições, a tarefa de realizar o diagnóstico das condições de saúde e de vida da comunidade e dos recursos disponíveis. Para esse fim, trabalha-se com o Sistema de Informação em Atenção Básica (SIAB), que facilita a obtenção de dados de forma sistemática sobre a saúde da população, permitindo assim identificar as condições de vida e adoecimento daquela comunidade.

A equipe mínima é composta por: um médico; um enfermeiro; de um a dois auxiliares de enfermagem; de quatro a seis agentes comunitários de saúde; um dentista; um técnico de higiene dentária e um auxiliar de cuidados dentários. Estes têm como uma das suas prioridades atuarem de forma integrada e com níveis de competência bem estabelecidos na abordagem da HAS.

\section{II - A Epidemiologia da HAS}

A HAS é uma doença crônica, multifatorial, em geral assintomática, e que exige tratamento contínuo e mudanças no estilo de vida, nem sempre fáceis de serem feitas. Ela atinge apro- 
ximadamente $15 \%$ da população adulta. Massie $(2001)^{4}$ constatou que $68 \%$ dos portadores da enfermidade desconhecem ter aumento dos seus níveis tensionais. Dos que estão diagnosticados, $53 \%$ fazem tratamento, e apenas $27 \%$ conseguem manter os níveis tensionais abaixo de 140/90 $\mathrm{mmHg}$.

Cabe ressaltar que há diferenças da prevalência de HAS nas populações, por influências biológicas, do estilo de vida, do ambiente, da organização do sistema de saúde e das possíveis interações entre os componentes desses elementos ${ }^{5}$. De acordo com o Sétimo Relatório Internacional do Joint National Committee sobre Prevenção, Detecção, Avaliação e Tratamento da Hipertensão Arterial ${ }^{6}$, a HAS afeta aproximadamente 50 milhões de pessoas nos EUA e 1 bilhão de pessoas no mundo.

No Brasil se desconhece a prevalência de HAS, havendo ainda dificuldades operacionais e metodológicas para a comparação de amplas variações encontradas em estudos de prevalências de HAS realizados por alguns autores entre as diferentes regiões brasileiras. Estes têm confirmado uma prevalência que varia entre $7,2 \%$ e $40,3 \%$ no Nordeste; 5,04 e $37,9 \%$ no Sudeste; $1,28 \%$ e $27,1 \%$ no Sul e 6,3\% e 16,75\% no Centro-Oeste. Como em outros países, a HAS e suas complicações geram elevados custos hospitalares, previdenciários, econômicos e sociais ${ }^{5}$.

Com o aumento da expectativa de vida e a diminuição da mortalidade por doenças infectocontagiosas, houve um aumento da prevalência das doenças crônico-degenerativas, como a HAS e o DM tipo 2, importantes fatores de risco para morbidade e mortalidade cardiovasculares. Em 1998, as doenças do aparelho circulatório foram responsáveis por $26,5 \%$ do total dos óbitos no Brasil, tendo como componentes principais as doenças cerebrovasculares (DCBV) e as doenças isquêmicas do coração (DIC) ${ }^{7}$.

\section{III - Avaliação e abordagem da pessoa com HAS}

O diagnóstico da HAS é feito por meio da identificação de aumento permanente dos níveis tensionais. O limite de normalidade aceitável é igual ou maior de 140/90 mmHg, em duas aferições, realizadas em momentos diferentes, em condições adequadas com método apropriado.

Recomendam-se os seguintes prazos para acompanhamento da pessoa com HAS. Essas orientações precisam ser individualizadas na prática clínica, levando-se em consideração as outras condições associadas à $\mathrm{HAS}^{8}$.

\section{Tabela I}

Recomendações para seguimento (prazos máximos)*

Pressão arterial inicial $(\mathrm{mmHg})^{* *}$

\begin{tabular}{|ccl|}
\hline Sistólica & Diastólica & \multicolumn{1}{c|}{ Seguimento } \\
$<130$ & $<85$ & Reavaliar em 1 ano \\
$130-139$ & $85-89$ & Reavaliar em 6 meses \\
$140-159$ & $90-99$ & Confirmar em 2 meses \\
$160-179$ & $100-109$ & Intervenção imediata ou \\
& & reavaliar em 1 semana \\
\hline
\end{tabular}

\footnotetext{
* Modificar o esquema de seguimento de acordo com a condição clínica do paciente.

** Se as pressões sistólica ou diastólica forem de categorias diferentes, o seguimento recomendado é definido como de menor tempo.

Fonte: III Consenso Brasileiro de Hipertensão Arterial, $1988^{8}$.
}

$\mathrm{Na}$ avaliação do paciente com hipertensão arterial, de acordo com o $7^{\circ}$ Relatório do Joint National 
Committe ${ }^{6}$, deve-se ter três objetivos principais:

1. Conhecer o estilo de vida do paciente e identificar outros fatores de risco cardiovasculares ou outros problemas concomitantes que possam afetar o manejo da HAS;

2. Identificar causas de HAS secundária (diagnóstico etiológico);

3. Verificar a presença ou a ausência de lesões de órgão alvo

Esses dados devem ser obtidos por meio da anamnese, do exame físico e dos exames complementares, quando indicados. A HAS então será classificada em grupos de acordo com os fatores de risco individual e os níveis pressóricos apresentados.

A abordagem da pessoa deve ser a do cuidado à pessoa adoecida, e não a uma doença, ou seja: uma abordagem que busque a parceria com a pessoa adoecida no seu cuidado de saúde e valorize o seu saber, promovendo a autonomia do indivíduo. Mesmo que a cura dessas patologias não seja possível, podemos trabalhar com o paciente o modo para alcançar uma vida com melhor qualidade e evitar as complicações incapacitantes como doenças cardiovasculares, insuficiência renal crônica, amputações e cegueira.

A adesão ao tratamento, farmacológico ou não-farmacológico, é um problema freqüente na abordagem desses pacientes. Sabe-se que, em média, dois terços dos pacientes com HAS não têm controle adequado dos níveis pressóricos. A maioria dos pacientes diagnosticados não adota mudanças suficientes no estilo de vida, não toma a medicação ou não faz uso de medicação suficiente para atingir o controle ${ }^{9}$.
Vários são os motivos que levam os pacientes a não aderirem ao tratamento. Esses podem ter relação com fatores diversos, os quais podem estar relacionados ao próprio indivíduo, tais como suas condições de vida, à relação médicopaciente, à equipe de saúde ou ao sistema de saúde.

Um aspecto importante para se alcançar a adesão ao tratamento é o vínculo da pessoa adoecida com a equipe de saúde, que deve estar preparada para dar suporte aos que demandam cuidados de saúde.

Os pacientes frequentemente alegam que não tomam a medicação porque "estão se sentindo bem". "Tratar por quê? Não sinto nada!" é uma frase que muitos dizem nas consultas. A dificuldade de cumprir as recomendações quanto a dietas e medicações são atribuídas à falta de recursos, por não entendimento da receita, pelos efeitos colaterais das drogas (impotência, tosse), por problemas familiares e no trabalho, às condições e ao estilo de vida.

A mudança no estilo de vida, muitas vezes suficiente por si só, para o controle da HAS, ou como coadjuvante para o sucesso da terapêutica medicamentosa, é difícil de ser alcançada. Esse problema é agravado pelo médico e outros profissionais de saúde, que não enfatizam esse aspecto do tratamento, subestimando a sua eficácia.

Segundo $\circ 7^{\circ}$ Relatório do Joint National Committee $^{6}$, a terapia mais efetiva prescrita por um clínico cuidadoso só controlará a HAS se o paciente estiver motivado, e a motivação é maior quando $\circ$ paciente tem experiências positivas $e$ confia no clínico. 


\section{IV - O Tratamento da pessoa com HAS}

O tratamento da HAS pode modificar a sua evolução, sendo os seus principais objetivos a redução das taxas de morbidade e mortalidade cardiovasculares e renais, proporcionando, conseqüentemente, uma melhor qualidade de vida às pessoas com HAS

A decisão do melhor tratamento para o indivíduo deve levar em conta, além dos valores da pressão arterial, a presença ou não de lesões em órgãos-alvo e de fatores de risco cardiovasculares associados. Consideram-se também as condições de vida da pessoa, as doenças associadas, o entendimento que elas têm do seu problema de saúde.

O Ministério da Saúde elaborou uma proposta de abordagem conjunta de HAS e DM na Atenção Primária à Saúde, já que são patologias com características semelhantes, como caráter crônico e necessidade de mudança do estilo de vida ${ }^{10}$. A abordagem do indivíduo com HAS se divide em duas linhas que se complementam.

Após a realização do diagnóstico, se identificada a necessidade de terapia medicamentosa, devemos traçar um plano terapêutico para aquela pessoa com os seguintes objetivos: ${ }^{10,11}$

- Conscientizar o paciente sobre a sua doença e necessidade do tratamento.

- Reduzir a PA a níveis normais com o mínimo de efeitos colaterais.

- Estar atento para a não-aderência ao tratamento.

- Manter tratamento simples e pouco dispendioso.

- Realizar a investigação necessária para afastar as causas secundárias.
- Utilizar o menor número de doses diárias necessárias.

- Adicionar uma droga por vez.

- Iniciar em pequenas doses.

- Avaliar os efeitos colaterais a partir do depoimento do paciente e ajustar a terapia valorizando a manutenção de uma boa qualidade de vida.

Neste trabalho, vamos dar ênfase à terapêutica não-medicamentosa, pois o nosso objetivo é demonstrar o valor desta abordagem para o sucesso do controle da hipertensão. A mudança no estilo de vida para prevenção de HAS é considerada indispensável para o controle das pessoas com hipertensão arterial.

O tratamento não-farmacológico inclui mudanças no estilo de vida e a adoção de hábitos mais saudáveis. A sua aplicação mostrou-se eficaz na redução da pressão arterial e na diminuição dos fatores de risco cardiovasculares.

Várias estratégias podem ser traçadas para conseguir que os pacientes atinjam essas mudanças no estilo de vida, dentre elas as atividades de grupo, uma abordagem por equipe interdisciplinar: médicos, enfermeiros, nuticionistas, psicólogos, dentre outros, e as atividades educativas.

\section{IV . 1 - Dieta}

Vários estudos epidemiológicos demonstraram a importância de uma dieta com restrição do sal. A resposta individual da HAS à variação da ingestão de sódio é muito variável. Indivíduos da raça negra, idosos e hipertensos ou diabéticos são mais sensíveis às alterações no consumo de sódio do que as outras pessoas da população geral ${ }^{10}$. 
O estudo epidemiológico colaborativo INTERSALT pesquisou a relação entre a pressão arterial e o consumo de sódio e de potássio, estudando também a relação da pressão arterial com outras variáveis, como peso, o índice de massa corporal e a ingestão de álcool. Foi planejado para identificar as relações dessas variáveis com a pressão arterial dentro de cada população e a comparação entre as diferentes populações estudadas. Concluiu-se, após análise de um conjunto de populações, que existe uma forte correlação entre a ingestão de sal e a pressão arterial e que, quanto mais alta a excreção urinária de sódio, mais alta é a pressão arterial ${ }^{12}$.

A título de curiosidade, ressalta-se que o estudo, analisando populações díspares, encontrou, por exemplo, que a população adulta dos índios Yanomami apresentou o menor nível de excreção de sódio até hoje relatado na literatura ${ }^{12}$.

\section{O ensaio clínico DASH (Dietary} Approaches to Stop Hypertension) estudou o efeito dos diferentes tipos de dieta na pressão arterial. Foram selecionados 459 indivíduos adultos com pressão arterial sistólica menor que $160 \mathrm{mmHg}$ e pressão arterial diastólica entre 80 e $95 \mathrm{mmHg}$, que foram submetidos a uma dieta típica dos americanos - rica em gorduras, com poucas frutas e verduras, durante três semanas. Após esse período, essas pessoas foram submetidas, durante oito semanas, a uma dieta controlada rica em frutas, verduras ou a combinação de uma dieta com frutas, verduras e pouca gordura. Nesta oportunidade, a ingestão de sal e o peso corporal eram controlados. Concluiu-se que a dieta rica em frutas e vegetais, com a redução do teor de gorduras, diminuiu os níveis pressóricos ${ }^{13}$.

A redução do consumo de alimentos ricos em gordura é uma orientação que deve ser dada ao paciente com hipertensão. Estudos epidemiológicos mostram que não existe efeito da variação do consumo de gorduras saturadas e não-saturadas com a pressão arterial, mas o consumo excessivo de gorduras contribui para outros fatores de risco cardiovasculares e para hipertensão e a obesidade.

O consumo excessivo de álcool é importante fator de risco para hipertensão arterial, além de poder causar resistência ao tratamento com medicações contra a hipertensão, sendo ainda fator de risco para $\mathrm{AVC}^{10}$. Recomenda-se que seja orientado às pessoas que consomem álcool limitar o consumo a não mais de $30 \mathrm{ml}$ de etanol/dia, (720 $\mathrm{ml}$ de cerveja, $300 \mathrm{ml}$ de vinho ou $60 \mathrm{ml}$ de uísque). Nas mulheres, por serem mais suscetíveis ao efeito do álcool, essa ingestão não deve ultrapassar $15 \mathrm{ml} / \mathrm{dia}$.

No INTERSALT se encontrou uma forte associação entre grande ingestão de álcool (mais de $300 \mathrm{ml}$ de álcool por semana) e a pressão arterial sistólica e diastólica. Outros estudos epidemiológicos mostram a relação do consumo de álcool com aumento da pressão arterial.

O estudo Pitt County é uma investigação longitudinal realizada desde 1988 com fatores preditores de HAS, com 970 americanos de origem africana, entre 25 e 50 anos de idade, que mostrou uma relação entre a dose de álcool consumida e a PA em homens e mulheres. Esse estudo mostrou aumento da PA em indivíduos que iniciaram o consumo de álcool durante o estudo. Outro dado importante desse estudo mostra que o aumento 
da PA em pessoas que pararam de beber é igual à de quem não bebia. Além disso, início e a continuidade do uso de álcool estão significativamente associados a fatores econômicos e sociais ${ }^{14}$.

\section{IV . 2 - Redução de Peso}

Os pacientes com HAS que tenham sobrepeso devem ser estimulados a participarem de uma estratégia de redução e controle do peso, pois existe uma relação direta com o aumento da pressão arterial, além de ser um fator de risco para as doenças cardiovasculares. Estudos epidemiológicos mostram que pacientes com IMC > $27 \mathrm{Kg}$ encontram-se com maior risco de desenvolverem DM tipo 2, HAS, dislipidemia e coronariopatia.

Nos Estados Unidos, há um aumento da prevalência da obesidade, sendo mais de um terço da população adulta obesa. Alguns estudos identificam a obesidade, o excesso de consumo de álcool e o envelhecimento como fortes preditores da incidência e prevalência de HAS. Outros estudos evidenciam a associação de obesidade com o desenvolvimento de HAS em pessoas mais jovens e desenvolvimento de HAS mais grave ${ }^{15}$.

No Brasil, $30 \%$ da população dos grandes centros urbanos apresentam excesso ponderal, e há também, como nos outros países, uma tendência crescente da prevalência da obesidade, tendo sofrido um incremento de $100 \%$ para os homens e $70 \%$ para as mulheres, em um período de 15 anos. Estudos evidenciam que a pobreza nos Estados Unidos é preditora de obesidade, e, em Porto Alegre, a obesidade é mais prevalente nas mulheres de classe econômica mais baixa, ocorrendo o contrário com os homens ${ }^{16}$. Ainda segundo Lessa (1998) ${ }^{16}$, dos elementos do campo de saúde que se associam à obesidade, os do estilo de vida são os mais importantes, pois dependem do indivíduo aceitar ou não as mudanças do dia-a-dia e de comportamentos nocivos à saúde. Outras variáveis importantes, como a raça negra e a pobreza, são preditores independentes de obesidade: ou são imutáveis (os biológicos, aos quais se acrescenta a herança e a idade) ou dependem de decisões políticas nacionais (as do ambiente socioeconômico), ante as desigualdades sociais, pelo menos no caso do Brasil.

A obesidade é definida como excesso de tecido gorduroso, e sua incidência vem aumentando nas duas últimas décadas. É também considerada uma doença da modernidade, havendo uma relação do aumento de sua incidência com certas características da sociedade moderna, como o sedentarismo e o alto consumo de alimentos ricos em gorduras. Havia uma dificuldade de se considerar a obesidade isoladamente como fator de risco para doenças cardiovasculares, pois geralmente há coexistência de outros fatores, como dislipidemia, DM e HAS.

Mais recentemente, algumas pesquisas demonstram que o maior valor preditivo para doença cardiovascular depende da forma de distribuição da gordura corporal. Há dados indicando que o depósito de gordura intraabdominal está relacionado com maior risco de coronariopatia.

Um dos resultados encontrados no INTERSALT foi uma significativa associação entre 
peso corporal dos indivíduos e pressão arterial sistólica e diastólica. Mais uma vez, pode ser citado - caso dos índios Yanomami, que não apresentaram aumento de peso com a idade, nem aumento dos níveis de tensão arterial ${ }^{13}$.

A diminuição de peso, mesmo que pequena, reduz a pressão em grande número de pessoas, além de aumentar o efeito das medicações anti-hipertensivas e reduzir os fatores de risco cardiovasculares existentes. Alguns estudos evidenciaram que a redução de peso é uma das medidas não-farmacológicas mais eficazes para redução dos níveis pressóricos ${ }^{10}$.

\section{IV.3 - Ingestão de potássio, cálcio e magnésio}

O paciente com HAS deve ter uma dieta rica em potássio, por meio da ingestão de alimentos como frutas e vegetais frescos. $O$ consumo elevado de potássio pode ter um efeito protetor contra o desenvolvimento de HAS e melhorar o controle da pressão arterial em hipertensos. A ingestão de quantidade inadequada de potássio pode aumentar a pressão arterial ${ }^{10}$.

Estudos epidemiológicos mostram a associação da dieta rica em potássio com diminuição dos níveis de pressão arterial. A excreção urinária de potássio foi inversamente associada com a pressão arterial sistólica e diastólica.

Os estudos epidemiológicos não evidenciaram os efeitos do consumo de cálcio na diminuição da pressão arterial, e outros estudos mostraram um pequeno efeito da suplementação de cálcio na redução da pressão. Não há, portanto, justificativas para se recomendar uso de suple- mento de cálcio com esse objetivo, tampouco dados convincentes que justifiquem recomendar o aumento do consumo de magnésio para diminuir a pressão arterial ${ }^{17}$.

\section{IV.4 - Exercícios físicos regulares}

A atividade física aeróbica regular pode colaborar na redução de peso, além de melhorar as condições de saúde, reduzir os fatores de risco cardiovasculares e de mortalidade por todas as causas de acordo ${ }^{10}$.

O benefício da atividade física para prevenção primária de HAS foi demonstrado em estudos epidemiológicos. O estudo Harvard Alumni incluiu 15 mil homens que não tinham HAS no ano de 1962 (PA < 160X95 mmHg). Em 1977, alguns desses indivíduos estavam hipertensos (PA > $160 \times 95 \mathrm{mmHg}$, critérios da época). Foi avaliado o tipo de atividade física que eles faziam (subir escadas, caminhada, algum tipo de esporte). Como resultado desse estudo foi observado nos indivíduos sedentários que o risco era $35 \%$ maior de desenvolver HAS do que nos indivíduos que realizavam algum tipo de atividade física ${ }^{18}$.

A atividade física é um componente central da abordagem não-farmacológica da HAS, com efeitos positivos tanto na saúde física quanto na mental. A combinação de atividade física regular e uma dieta adequada (baixa ingestão de gorduras e açúcares, rica em vegetais, frutas, grãos e peixes) contribui para a diminuição do risco de desenvolver obesidade e outros benefícios para a saúde como a redução dos níveis pressóricos.

O sedentarismo é um dos principais fatores de risco para as cardiopatias isquêmicas, contribui também para outros fatores de risco, como exem- 
plo, obesidade e HAS, dentre outros, e aponta a atividade física regular como benéfica para o controle desses fatores de risco ${ }^{18}$.

O Centro de Controle de Doenças (CDC) e o American College of Sports Medicine ${ }^{19}$ recomendam que:

- Toda pessoa com mais de dois anos de idade deve acumular pelo menos 30 minutos de atividade física de intensidade moderada no maior número de dias da semana (preferencialmente todos os dias);

- Benefícios adicionais à saúde podem ocorrer com o aumento da quantidade de tempo de exercício e da intensidade da atividade;

- Pessoas com cardiopatia sintomática, diabetes mellitus ou outras doenças crônicas devem ser avaliadas por um médico e receber um programa de exercício apropriado para a sua situação;

- Homens sedentários com mais de 40 anos, mulheres com mais de 50 anos ou pacientes com alto risco de cardiopatia devem procurar um médico antes de começarem um programa de atividade física;

- Atividades para desenvolver resistência e força devem ser realizadas duas vezes na semana. É necessário, porém, cuidado com o perigo de atividades físicas vigorosas em pessoas sedentárias ou com hipertensão não-controlada, ou que tenham risco para eventos cardiovasculares como fibrilação atrial, angina e morte súbita.

\section{IV . 5 - Tabagismo}

Uma pesquisa realizada em dez capitais brasileiras, em 1988, mostrou que o consumo de cigarro é maior nas classes de menor rendimento, e atribuiu-se a essa condição o fato da população economicamente mais pobre ter menos acesso a informações do risco desse hábito. Notou-se também que, nessa população, uma parcela grande dos rendimentos é usada para o consumo de cigarros, em detrimento de outros itens necessários para a vida, como, por exemplo, a alimentação. O consumo maior de cigarro e as outras condições às quais essa população é submetida - como desnutrição, doenças infecciosas e do trabalho - levam a um adoecimento mais frequente e, conseqüentemente, a uma piora progressiva da qualidade de vida dessas pessoas $^{20}$.

O tabagismo é um poderoso fator de risco não só para as doenças cardiovasculares, como para doenças pulmonares e câncer, sendo fundamental a orientação da interrupção desse hábito.

Existem métodos que auxiliam na abordagem do tabagismo, como, por exemplo, método comportamental, medicamentoso e outros (acupuntura, hipnose, etc.). O Instituto Nacional do Câncer elaborou um material para que o profissional de saúde possa auxiliar o seu paciente a parar de fumar por meio desses métodos. 0 método comportamental foi indicado como o mais apropriado para ajudar o fumante a parar de fumar, pois abandonar esse hábito é um processo, e, na maioria das vezes, leva tempo, pois exige adaptações a um novo estilo de vida. O paciente deve ser estimulado a parar de fumar independente do número de tentativas fracassadas ${ }^{21}$.

Há relatos na literatura de que a PA e a freqüência cardíaca se elevam com o ato de fumar 
e que as pessoas com hipertensão que continuam a fumar podem não obter toda a proteção contra as doenças cardiovasculares oferecida pelo tratamento com medicações anti-hipertensivas.

Epidemiologicamente, a relação entre o tabagismo e a hipertensão é freqüentemente indefinida devido à associação dos fatores de risco de ambos. É mais freqüente entre os fumantes um elevado consumo de álcool e pequeno consumo de vegetais e frutas, fatores esses que estão associados à hipertensão.

\section{IV.6 - Relaxamento e Biofeedback}

O estresse emocional eleva, de forma aguda, a pressão arterial, mas o papel das técnicas de relaxamento ainda é incerto. Estudos epidemiológicos mostraram pouco efeito além daquele observado em grupos-controle. Portanto, a literatura disponível não corrobora o uso de técnicas de relaxamento para o tratamento definitivo ou para a prevenção de hipertensão ${ }^{10}$.

\section{V - Conclusão}

Após a análise de todos esses estudos apresentados, concluímos que há fortes evidências da eficácia do tratamento não-farmacológico na abordagem das pessoas com HAS. Com a prática de exercícios físicos, uma alimentação saudável e a redução do peso corporal, podem se alcançar um melhor controle dos níveis pressóricos, podendo assim evitar as complicações da HAS. Com a interrupção do tabagismo, é retirado um dos principais fatores de risco, não só para doenças cardiovasculares.

Essas evidências fornecem substratos suficientes para a elaboração de políticas públicas de saúde que interfiram na incidência e nas conseqüências da HAS na população.

Um dos estudos apresentados evidenciou a associação da pobreza com o tabagismo, a obesidade e, conseqüentemente, a HAS, identificando, com isso, a necessidade de criação de estratégias que melhorem as condições de vida da população e possibilitem as pessoas com HAS terem condições de fazer algum tipo de atividade física, ter uma alimentação saudável e o controle adequado do peso.

A Estratégia de Saúde da Família pode colaborar para o favorecimento dessas condições, pois tem como um dos seus objetivos possibilitar a identificação de recursos na comunidade para execução de ações que melhorem as condições de vida da comunidade.

Outra questão identificada foi a dificuldade para a determinação da prevalência de HAS no Brasil, que também poderá ser mudada com a ESF - especialmente na medida em que esta aumenta a cobertura de população por ela assistida. Dessa forma, teremos mais acesso às informações de saúde obtidas pela equipe de saúde da família, por meio do Sistema de Informações da Atenção Básica (SIAB).

\section{VI - Referências}

1- Joseph B, Muhlestein MD. Risk factor modification for cardiac disease. Chronic infection and coronary artery disease. Med Clin North Am 2000; 84 (1): 267-77.

2-Souza e Silva NA, Oliveira GMM, Klein CH. Mortalidade por doenças do aparelho circulatório do Estado do Rio de Janeiro. [s.l.: s.n.]; [s.d.].

3 - Brasil. Ministério da Saúde. Departamen- 
tamento da Atenção Básica. Guia prático do programa Saúde da Família. Brasília (DF): Ministério da Saúde; 2001.

4- Massie BM. Systemic Hypertension. In: Tierney ML, McPhee SJ, Papadakis MA. Current Medical Diagnosis and Treatment. $40^{\text {th }}$ ed. [s.I.]: Lange Medical Books/McGraw-Hill; 2001. p.448-72.

5- Lessa I. Epidemiologia da hipertensão. In: Lessa I. O adulto brasileiro e as doenças da modernidade- epidemiologia das doenças crônicas não transmissíveis. São Paulo, Rio de Janeiro: HUCITEC, ABRASCO; 1998. p. 77-96.

6- The Joint National Committee on Prevention, Detection, Evaluation, and treatment of high blood pressure. The seventh report of the Joint National Committee on Prevention, Detection, Evaluation, and Treatment of High Blood Pressure. JAMA. 2003 May; 289: 2560-72.

7- Brasil. Ministério da Saúde. Citado por Sousa e Silva NA, Oliveira GMM, Klein $\mathrm{CH}$. Mortalidade por doenças do aparelho circulatório do Estado do Rio de Janeiro.

8- III Consenso Brasileiro de Hipertensão Arterial. Rev Bras Clin Terap 1988;24(6)231-272.

9- The Joint National Committee on Prevention, Detection, Evaluation, and treatment of high blood pressure. The sixth report of the Joint National Committee on Prevention, Detection, Evaluation, and Treatment of High Blood Pressure. Arch Intern Med 1997; 157:2413-46.

10- Brasil. Ministério da Saúde. Departamento de Ações Programáticas Estratégicas. Plano de reorganização da atenção básica à hipertensão arterial e ao diabetes mellitus: manual de hipertensão arterial e diabetes mellitus. Brasília (DF): Ministério da Saúde; 2001.

11 - Silveira CAM, Remígio MI, Brandão S. Hipertensão Arterial Sistêmica. In: Filgueira NA, Costa Júnior JI, Leitão CCS, Lucena VG, Melo HRL, Brito CAA. Condutas em Clínica Médica. 2. ed. Rio de Janeiro: MEDSI; 2001. p. 314-32.

12- Souza e Silva NA, Mancilha-Carvalho JJ. Os Yanomami no INTERSALT. Arq Bras Cardiol. 2003; 80(3):289-94.

13- Appel LJ, Moore TJ, Obarzaneck E, Vollmer WM, Svetkey LP, Sackes FM, et al. A clinical trial of the effects of dietary patterns on blood pressure: DASH Collaborative Reserch Groups. N Engl J Méd. 1997; 336:1117-24.

14- Curtis AB, James AS, Strogatz DS, et al. Alcohol comsuption and changes in blood pressure among African Americans: The Pitt County Study. Am J Epidemiol 1997; 146:727-33. Citado por: Labarthe D, Ayala C. Nondrug interventions in hypertension prevention andepidemiologia das doenças crônicas não transmissíveis. São Paulo, Rio de Janeiro: HUCITEC, ABRASCO; 1998. p. 13953. control. Cardiol Clin 2002 May; 20(2): 249-63.

15- Paffenbager Jr. RS, Hyde RT, Wing AL, et al. Physical activity and incidence of hypertension in college alumni. Am J Epidem 1983; 117:245-57. Citado por: Labarthe D, Ayala C. Nondrug interventions in hypertension prevention and control. Cardiol Clin 2002 May; 20(2): 249-63.

16- Lessa I. Obesidade. In: Lessa I. O adulto brasileiro e as doenças da modernidade.

17 - Labarthe D, Ayala C. Nondrug interventions in hypertension prevention and control. Cardiol Clin. 2002 May; 20(2): 249-63.

18- Thompson PD et al. Exercise and 
Physical Activity in the Prevention and Treatment of Atherosclerotic Cardiovas- cular Disease. Circulation 2003 June; 24.

19- CDC. Physical activity and Heath: A Report of the Surgeon General. Executive Summary. Disponível em: http://www.cdc.gov/nccdphp/sgr/ pdf/execsumm.pdf

Acesso em 19/07/2006.

20- Brasil. Ministério da Saúde. Instituto Nacional de Câncer. Coordenação Nacional de Controle do Tabagismo e Prevenção Primária de Câncer. Falando sobre tabagismo. 3 ed. Rio de Janeiro: Instituto Nacional do Câncer; 1998.

21- Brasil. Ministério da Saúde. Instituto Nacional de Câncer. Coordenação Nacional de Controle do Tabagismo e Prevenção Primária de Câncer. Ajudando seu paciente a deixar de fumar. Rio de Janeiro: Instituto Nacional do Câncer; 1997.

Endereço de Correspondência:

Carolina Fajardo

Rua Conde de Baependi 70/402

CEP 22231140

Rio de Janeiro RJ

Endereço eletrônico:

carolfajardo@yahoo.com.br 